\title{
LINFOMA PRIMARIO DE SISTEMA NERVIOSO CENTRAL EN PACIENTE INMUNOCOMPETENTE. REPORTE DE UN CASO
}

\author{
${ }^{1}$ Loria Ávila, Esteban; ${ }^{2}$ Granados Brenes, Gabriela y ${ }^{3}$ Rojas Mena, Betzabé
}

${ }^{1}$ Médico general, Hospital Dr. Rafael Ángel Calderón Guardia, San José, Costa Rica

${ }^{2}$ Médico asistente y Especialista en hematología, Hospital Calderón Guardia, San José, Costa Rica

${ }^{3}$ Médico Especialista en anatomía patológica, Hospital Calderón Guardia, San José, Costa Rica

Resumen: El linfoma no Hodgkin (LNH) primario de Sistema nervioso central (SNC), (LPSNC) es una rara entidad que se define por la presencia de lesiones en SNC sin evidencia de tumor primario al momento de su diagnóstico. Objetivos: describir un caso de LPSNC, y realizar una revisión bibliográfica sobre el tema. Mujer de 56 años inmunocompetente, que consultó por síndrome confusional. Se determinan 2 lesiones a nivel de cerebro mediante TAC, sin lesiones en otras partes del cuerpo, y se realizó biopsia de dicha tumoración, confirmando LNH de células B, por lo que se inició protocolo DeAngelis con adecuada evolución.

Palabras clave: Linfoma, Sistema Nervioso Central 


\title{
PRIMARY CENTRAL NERVOUS SYSTEM LYMPHOMA IN AN IMMUNOCOMPETENT PATIENT. CASE REPORT
}

\begin{abstract}
Primary central nervous system non-Hodgkin's lymphoma (PCNSL) is a rare form of non-Hodgkin lymphoma (NHL) characterized by the presence of injuries to the CNS and no evidence of a primary tumor in the initial diagnosis. Objectives: To describe a PCNSL case and to do a bibliographical review on the subject. The patient is a 56 year old immunocompetent woman, who consulted the doctor about a confusional syndrome. Whole body CAT scan only showed two brain injuries. A B-cell NHL was found after the tumor's biopsy, and therefore the DeAngelis protocol was started; the results obtained were satisfactory.
\end{abstract}

Key words: Lymphoma, Central Nervous System

\section{INTRODUCCIÓN}

El LPSNC comprende el $1 \%$ de todos los linfomas y el $4 \%$ de todos los tumores cerebrales primarios. La mayoría son esporádicos, mientras que en una minoría están asociados con inmunodeficiencia congénita o adquirida. La incidencia de LPSNC está aumentando tanto en inmunocompetentes y en pacientes portadores del virus de inmunodeficiencia humana (VIH) [1].

\section{REPORTE DE CASO}

Femenina de 56 años, conocida sana, referida a psiquiatría por cambios de comportamiento de tres meses de evolución, pérdida de la memoria inmediata y desorientación. Posteriormente asoció letargia, cefalea y mareos. Niega fiebre y pérdida de peso. Se decide descartar organicidad, se realiza un TAC de cráneo, y se describe "lesión redondeada frontal izquierda, la cual oblitera parcialmente el cuerno anterosuperior del ventrículo lateral izquierdo con importante edema alrededor. Se observa otra lesión redondeada paramedial, parietal derecha con importante edema perilesional" (Ver Figura 1).
La paciente fue valorada por neurocirugía, y ante la presencia de lesiones múltiples, se pensó como primera posibilidad que se tratara de metástasis a sistema nervioso central y se solicitó valoración a oncología médica para determinar el origen del tumor primario.

Se realizaron múltiples estudios y no se logró encontrar enfermedad en otros órganos por lo que se solicitó una biopsia de cerebro.

Se tomó una biopsia de la masa frontal y se reportó como Linfoma No Hodgkin difuso de células grandes B (Ver figura 2).

Descripción de biopsia: figura 2.A. Parénquima cerebral infiltrado por una neoplasia de estirpe linfoide figura 2.B. Caracterizada por células de tamaño grande, núcleos vesiculosos, con múltiples nucléolos, escaso citoplasma y abundantes mitosis. Se distribuyen predominantemente de forma angiocéntrica, y formando sábanas, con linfocitos T CD3 positivos acompañantes. Con técnicas de inmunohistoquímica figura 2.C. y 2.D. Las células neoplásicas expresan CD20 y MUM-1 focal, son negativas para CD3, CD10, bcl-6, bcl-2, CD-5, con un índice de proliferación (Ki-67) del 70\%. Diagnóstico: Linfoma Difuso de Células Grandes B. 


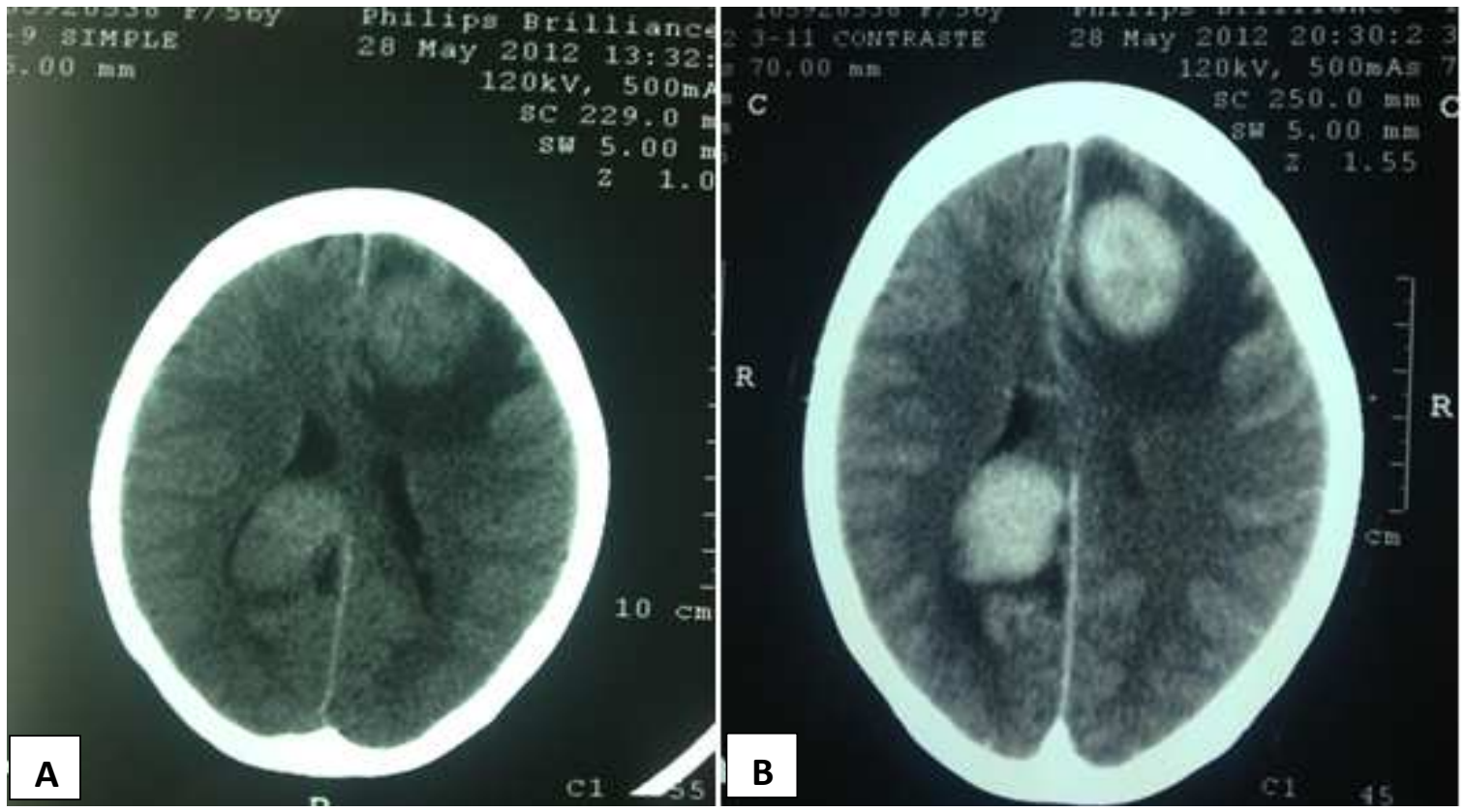

Figura 1. A. TAC de cerebro sin contraste. B. TAC de cerebro con contraste. Descripción de TAC: Se observan 2 lesiones ocupantes de espacio las cuales en la figura 1.B realzan intensamente con el medio de contraste y generan importante edema vasogénico perilesional la frontal izquierda alcanza los $29 \mathrm{~mm}$ y la parietal derecha $30 \mathrm{~mm}$ de diámetro.

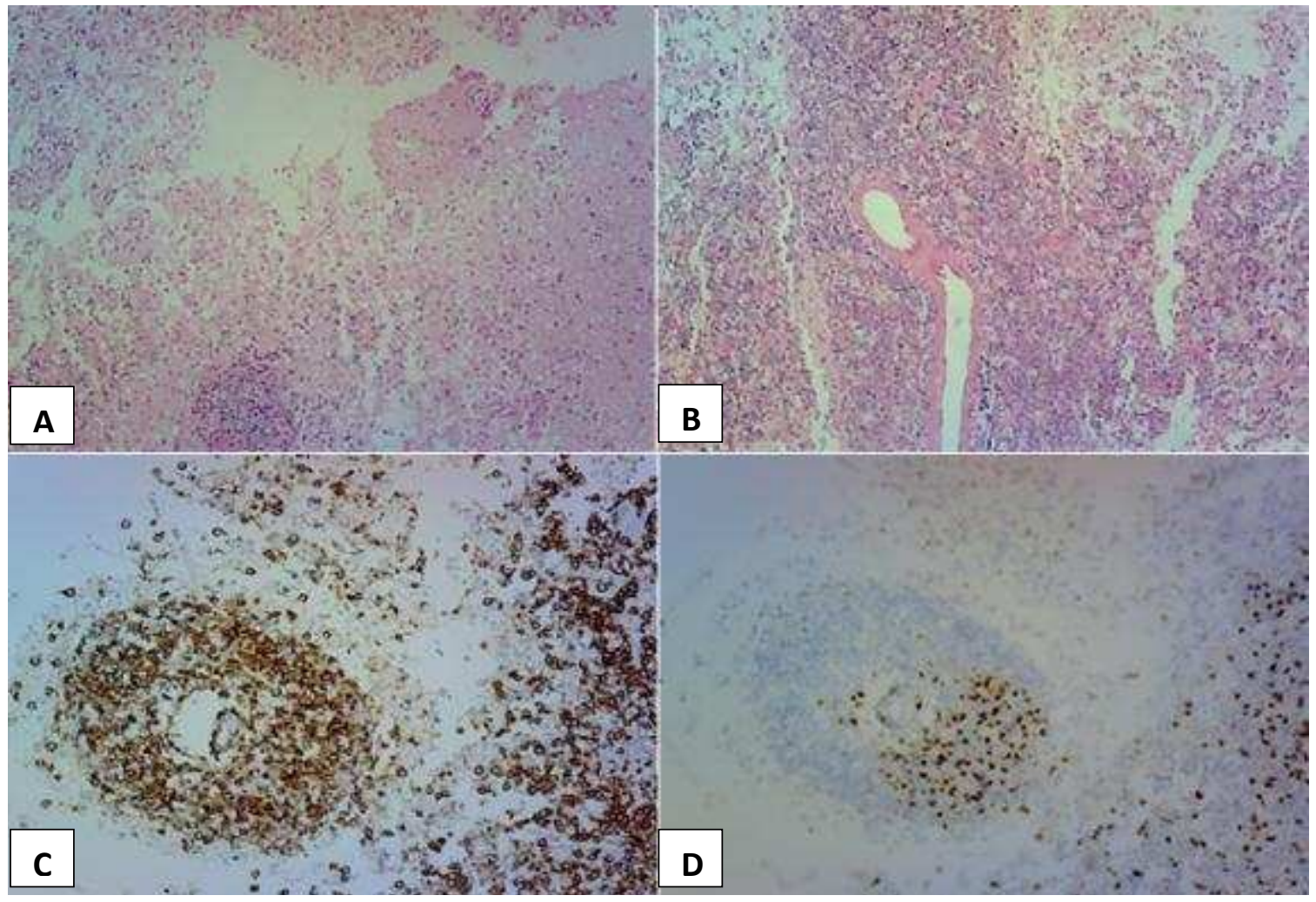

Figura 2. A. Hematoxilina-eosina Parénquima cerebral y Linfocitos B neoplásicos (*). B. Hematoxilina-eosina, angiocentricidad. C. Inmunohistoquímica CD 20 linfocitos neoplásicos. D. Inmunohistoquímica CD 3 linfocitos T acompañantes.

Revista electrónica publicada por el Departamento de Farmacología de la Escuela de Medicina de la Universidad de Costa Rica, 2060 San José, Costa Rica. ${ }^{\circledR}$ All rights reserved. Licensed under a Creative Commons Unported License. 
Tres semanas después de la biopsia ingresó al servicio de emergencias por hemiparesia derecha y afasia de Broca de $48 \mathrm{~h}$ de evolución.

En emergencias se valoró por hematología, se determina que no hay lesiones en tórax o abdomen, se toma una biopsia de médula ósea, la cual no mostró infiltración y una serología por HIV que se reportó negativa. Líquido cefalorraquídeo se encontraba negativo por infección o infiltración neoplásica.

Se decidió iniciar tratamiento con protocolo De Angelis, (Sin procarbazina, que no está disponible en el país).
A las 48 horas del inicio del tratamiento la paciente mejoró de la afasia y de la hemiparesia y una semana después ya caminaba sin ayuda y hablaba normalmente.

Se completó esquema de tratamiento con quimioterapia y radioterapia y se revaloró con resonancia magnética (ver figura 3) considerándose en remisión completa. Como única secuela epilepsia lesional, controlada con anticonvulsivantes.

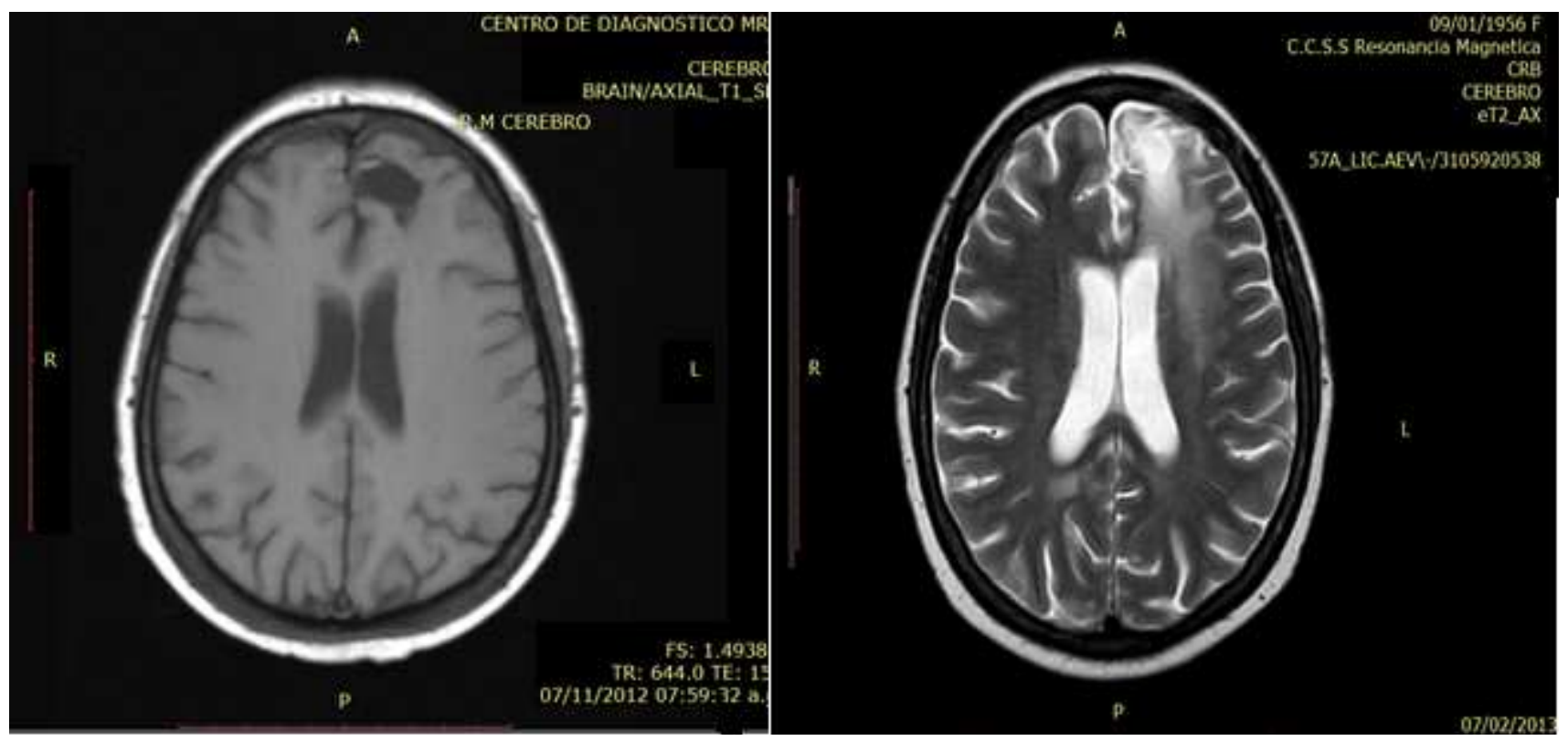

Figura 3. A. Resonancia magnética de cerebro Corte axial T1 B. Resonancia magnética de cerebro. Corte axial T2. Descripción de RM: figura 3.A. se aprecia un nódulo en el interior de formación hiperdensa en secuencia de T1, figura 3.B. Existe intensidad de la sustancia blanca a su alrededor que se extiende atreves de todo el lóbulo izquierdo. Sin signos de evidente recidiva tumoral y los cambios podrían estar dados por encefalomalacia y patrón de radionecrosis y gliosis.

\section{DISCUSIÓN}

El linfoma primario de sistema nervioso central (PSNCL) es una rara forma de linfoma no Hodgkin, poco frecuente y de características agresivas. A menudo compromete las leptomeninges, el ojo y raramente el cordón espinal [2].

Revista electrónica publicada por el Departamento de Farmacología de la Escuela de Medicina de la Universidad de Costa Rica, 2060 San José, Costa Rica. ${ }^{\circledR}$ All rights reserved. Licensed under a Creative Commons Unported License. 


\section{EPIDEMIOLOGÍA}

Representa menos del 1-2\% de todos los linfomas no Hodgkin y 3-6\% de todos los tumores primarios de cerebro [3].

Se ha relacionado con pacientes inmunosupresos (HIV) [1]. La estirpe histológica más frecuente de los PSNCL es el linfoma difuso las células grandes B 90\%, el otro 10\% suele verse en linfoma de Burkitt, linfoma de bajo grado, linfomas de células T1 [4].

\section{FISIOPATOLOGÍA.}

Los linfomas de SNC surgen de centros germinales tardíos o centros postgerminales se ha determinado que los genes involucrados en la diferenciación, proliferación, apoptosis se encuentran implicados en la linfoproliferación y tropismo de estas células por el microambiente del sistema nervioso central, se observó que las células tumorales estimulan los astrocitos y células de la microglia las cuales expresan citoquinas y receptores para citoquinas que permiten la penetración de las células tumorales a través de las barrera hematoencefalica y la diseminación intracerebral [5].

\section{PRESENTACIÓN CLÍNICA}

Se puede presentar en cualquier edad, la media se encuentra entre 53-61 años, predomina levemente en el sexo masculino, con una presentación de una masa la mayoría de las veces a nivel supratentorial $[4,6]$.

La duración de los síntomas persiste por 2 o 3 meses antes de que el paciente consulte por su sintomatología, los cuales son debidos a aumento de la presión intracraneal, sintomatología neuropsiquiátrica (los cambios cognitivos y de personalidad son los más frecuentes, reflejando su predilección por los lóbulos frontales, el cuerpo calloso y las estructuras periventriculares) [7]. Además déficit neurológico focal, convulsiones, perdida de la visión, dolor retroocular, el paciente refiere ocasionalmente la presencia de síntomas $\mathrm{B}$ fiebre, pérdida de peso y diaforesis nocturna $[5,8,14]$.

\section{DIAGNÓSTICO.}

Consiste de un examen físico neurológico, búsqueda de adenopatías, examen testicular en hombres, evaluación psiquiátrica, citología citometría, cuantificación de inmunoglobulinas, RM con gadolinio, TAC con medio de contraste, descartar foco primario con TAC de tórax y abdomen, aspirado de biopsia, examen oftalmológico con el fin de descartar afección del nervio óptico, retina y cavidad vítrea. Se debe al momento del abordaje solicitar DHL, hemograma completo, aclaramiento de creatinina, serología HIV $[7,9,10]$.

Las imágenes han permitido hacer el diagnóstico de una manera más precisa y temprana. Aunque se prefiere e uso de resonancia magnética desde la presentación de los síntomas neurológicos, debido a que se facilita el diagnóstico de presentaciones atípicas $o$ de lesiones infiltrativas, no se puede obviar el uso del TAC para diagnóstico de la lesión $[6,4,10]$.

La mayoría de las veces es posible observar una lesión espacio ocupante que se encuentre en contacto con el espacio subaracnoideo y sin la necrosis característica [7].

Los corticoesteroides deben ser evitados antes de la toma de la biopsia, porque puede interferir con el diagnóstico. La dexametasona es asociada con una respuesta inicial del $70 \%$ aunque transitoria [10]. La desaparición de la lesión no debe ser considerada como diagnóstica ya que también la sarcoidosis, esclerosis múltiple, encefalomielitis aguda y otros tumores también pueden responder dramáticamente a los esteroides [11].

Se ha visto también que el uso de la 18 fluoro-deoxiglucosa mediante el PET provee una sensibilidad de $75-87 \%$ en el diagnóstico de lesiones infiltrativas nerviosas más pequeñas 
comparado con los métodos convencionales de TAC y RM, pero es un método muy poco sensible para determinar lesiones infiltrativas intraoculares $[4,7]$.

La biopsia estereotáctica es el procedimiento estándar para proveer una muestra para el diagnóstico histopatológico [12].

La inmunohistoquímica es uno de los métodos más importantes utilizados para la clasificación de linfomas. Se utilizan como marcadores el CD20 para la estirpe de linfoma de células B [13]. La actividad proliferativa del tumor es medida por la expresión del antígeno Ki-67, la cual vincula la tasa de proliferación del tumor y representa un índice pronóstico reconocido en diversos tumores. Mediante la técnica de inmunohistoquímica es posible establecer el recuento celular en un campo de alto poder y de esta manera establecer la tasa de proliferación del tumor [14].

\section{TRATAMIENTO}

En pacientes no tratados el pronóstico es malo, con una sobrevida media de 3 a 4 meses. La radioterapia sola usualmente resulta en una sobrevida de aproximadamente 12 meses y en pacientes mayores de 60 años la media es solo de 7 meses. La combinación de radioterapia y quimioterapia ha mejorado la sobrevida a 30-60 meses [15].

Se ha reconocido que el uso de dosis altas de metrotexate es el tratamiento que ha optimizado y ha tenido un impacto en el mejoramiento de la sobrevida de los pacientes. A dosis de $2,5 \mathrm{mg} / \mathrm{m}^{2}$ alcanza concentraciones terapéuticas en el líquido cefalorraquídeo especialmente cuando se administra en una infusión rápida de dos o tres horas [16].

El metrotexate puede ser administrado además por vía intratecal, en este caso se ha visto una disminución de diez veces la concentración a nivel ventricular si el paciente no se mantiene en posición prona por al menos una hora después de la aplicación [17].

El uso de radioterapia en rangos de 20-55 Gy y se ha observado un incremento en la proporción de sobrevida de los pacientes, pero también se ha documentado el impacto de esta por su neurotoxicidad, el uso concomitante de quimioterapia $y$ radioterapia ha mostrado remisión completa cuando esta se usa después de la quimioterapia $[7,8,18]$.

La cirugía es restringida únicamente a la obtención de material histopatológico que permita establecer el diagnóstico. Cualquier intento de remoción del tumor se encuentra totalmente contraindicado, debido a que la evidencia de la remoción total o parcial empeoraría significativamente la sobrevida del paciente $[8,9,19]$.

\section{EFECTOS ADVERSOS POS TRATAMIENTO}

Los tratamientos utilizados en el PCNSL han mostrado su alta capacidad de neurotoxicidad, se ha evidenciado que el rol de la quimioterapia y la radioterapia, tienen una alta correlación entre las disfunciones cognitivas (atención, memoria, funciones ejecutorias, habilidades psicomotoras $[6,8,14]$.

\section{CONCLUSIÓN}

El LPSNC en pacientes HIV negativos es una enfermedad sumamente infrecuente. En el caso presentado, se estudio la paciente, por presentar clínica de alteración de la conciencia en el cual se documentan mediante TAC, dos masas a nivel de $\mathrm{SNC}$, se ingresa por sospecha de tumor primario por lo que al descartarlo, finalmente se realiza biopsia y se obtiene el diagnóstico histopatológico de linfoma de SNC de células grandes B, con adecuada evolución al protocolo de DeAngelis, se documento al final del tratamiento sin recidivas por medio del reporte de resonancia magnética.

Revista electrónica publicada por el Departamento de Farmacología de la Escuela de Medicina de la Universidad de Costa Rica, 2060 San José, Costa Rica. ${ }^{\circledR}$ All rights reserved. Licensed under a Creative Commons Unported License. 
Por lo que teniendo en cuenta lo infrecuente de la patología se logro establecer un adecuado abordaje multidisciplinario, el cual al descartar el tumor primario y establecer mediante la biopsia de SNC, el diagnóstico que permitió un inicio del tratamiento y resolver la patología que la paciente presento. A pesar de lo infrecuente de la patología un abordaje temprano de los linfomas en SNC, permite una evolución satisfactoria en este tipo de tumores a nivel de SNC en el que su tratamiento es médico y no quirúrgico.

\section{AGRADECIMIENTOS}

Fernández Vieto, Jaime. Médico asistente y especialista en radiología Hospital Calderón Guardia, por su ayuda en la descripción de la TAC.

Esquivel Solís, Faylan. Médico asistente y especialista en radiología, del centro nacional de Resonancia Magnética Hospital Calderón Guardia por su ayuda en la descripción de la RM.

\section{REFERENCIAS}

1. Kristina M. Braaten, Rebecca A. Betensky, de Leval, Laurence, Okada, Yoshifumi Hochberg, Fred H. Louis, David N. Harris, Nancy L. and Batchelor Tracy T. BCL-6 Expression Predicts Improved Survival in Patients with Primary Central Nervous System Lymphoma. Clinical Cancer Research 2003 Mar; 9: 1063-1069.

2. DeAngelis LM, Yahalom J: Primary central nervous system lymphoma, in DeVita V (ed) Cancer: Principles and Practice of Oncology. Philadelphia, Lippincott Williams \& Wilkins, 2001, pp: 22302232.

3. U.S. cancer statistics working group. united states cancer statistics: 1999-2004 incidence and mortality web-based report. atlanta:U.S. department of health and human services, centers for disease control and prevention and national cancer institute. 2007 [cited 1/22/2008]. Available

from:http://apps.nccd.cdc.gov/uscs/Table.aspx?G
roup=TableAll \&Year=2004\&Display $=$ n. accessed Dec 12, 2009.

4. Priscilla K. Brastianos,Tracy T. Batchelor. Primary Central Nervous System Lymphoma Overview of Current Treatment Strategies. Hematol Oncol Clin N Am 2012 Aug; 26: 897-916.

5. Carole Soussaina, Khe Hoang-Xuan. Current Primary central nervous system lymphoma: an update. Opinion in Oncology 2009 Nov; 21:550558.

6. Y.Z. Tang, T.C. Booth, P. Bhogal, A. Malhotra, T. Wilhelm.Imaging of primary central nervous system lymphoma. Clinical Radiology 2011 Mar, 66: 768-777.

7. Lister Andrew, Abrey Lauren, Sandlund John T. Central Nervous System Lymphoma.American Society of Hematology. Hematology 2002. p283296.

8. Brian J. Scott, Vanja C. Douglas, TarikTihan, James L. Rubenstein, S. Andrew Josephson. A Systematic Approach to the Diagnosis of Suspected Central Nervous System Lymphoma. JAMA Neurol 2013 Jan;70(3):311-319.

9. Schultz, christopher j., bovi, Joseph. Current management of primary central nervous system lymphoma, Int. J. Radiation Oncology Biol Phys 2010 Mar; 76(3), pp. 666-678.

10. Mohile, Nimish A. Abrey, Lauren E. Primary Central Nervous System Lymphoma. Neurol Clin 2007 Nov; 25: 1193-1207.

11. Ferreri.Andre's J. M.How I treat primary CNS lymphoma. Blood 2011 Jul; 118(3):510-522.

12. Slaviero, Felipe, Frighetto, Leonardo, Casali, JairoJúnior, Santos, MoemaNenê, Vanzin, José Ricardo, D. AzambujaJr, Nério Primary Central Nervous system Lymphoma in an inmunocompetent host.. Arq Neuropsiquiatr 2008 Set; 66(3-A):542-544.

13. Qing-ping Jiang, Shao-yan Liu, Yue-xin Yang, Xuexian Tan, Juan Peng, Zhong-tang Xiong, and Zhi Li. CD20-positive NK/T-cell lymphoma with indolentclinical course: report of case and review ofliterature. Diagn Pathol 2012 Oct; 7: 133.

14. Szczuraszek, Katarzyna, Mazur, Grzegorz, Jeleń, Michał, Dzięgiel, Surowiak, Piotr Paweł and Zabel, Maciej, Prognostic Significance of Ki-67 Antigen

Revista electrónica publicada por el Departamento de Farmacología de la Escuela de Medicina de la Universidad de Costa Rica, 2060 San José, Costa Rica. ${ }^{\circledR}$ All rights reserved. Licensed under a Creative Commons Unported License. 
expression in Non-Hodgkin's Lymphomas. Anticancer Research 2008 Mar-abr; 28:11131118.

15. Ney DE, Reiner AS, Panageas KS, Brown HS, DeAngelis LM, Abrey LE. Characteristics and Outcomes of Elderly Patients with Primary Central Nervous System Lymphoma. Cancer 2010 Oct 1; 116(19):4605-12.

16. DeAngelis, Lisa M.,Seiferheld, Clifford, Wendy S. Schold, Fisher, Barbara and Schultz,Christopher J. Combination chemotherapy and radiotherapy for primary central nervous system lymphoma: radiation therapy oncology group study 93-10. Journal of Clinical Oncology 2002 Dec 15; 20(24); 4643-4648.

17. Doucet, PStephane, Kumthekar, Priya, RaizerrimaryJeffrey. PrimaryCentral Nervous System Lymphoma. Current Treatment Options in Oncology 2013Mar, DOI 10.1007/s11864-0130227.

18. Pui,Ching-Hon, Thiel, Eckhard. Central Nervous System Disease in Hematological Malignancies: Historical Perspective and Practical Applications. Semin Oncol 2009 Aug; 36(4 Suppl 2): S2-S16.

19. Pels, Hendrik, Schlegel, Uwe. Primary Central Nervous System Lymphoma. Current Treatment Options in Neurology 2006 Jul; 8:346-357.

\section{INFORMACION DE AUTOR:}

Loria Ávila, Esteban

robertesteban@gmail.com 